\title{
Dynamische geometrische Konturmodelle zur Segmentierung intravaskulärer Ultraschallbildfolgen für die dreidimensionale Oberflächenrekonstruktion koronarer Gefäßwände
}

\author{
Schmauder M. ', Orglmeister R..', Volk D. ${ }^{1}$, Aldekamp F.', Gross C.M. ${ }^{2}$, Waigand J. ${ }^{2}$ \\ ${ }^{1}$ Technische Universität Berlin, Institut für Elektronik und Lichttechnik, EN-3, D-10587 Berlin \\ ${ }^{2}$ Universitätsklinikum Charité, Medizinische Fakultät der Humboldt-Universität zu Berlin, \\ Campus Berlin-Buch, Franz-Volhard-Klinik am Max Delbrück Centrum
}

\section{EINLEITUNG}

Die minimal invasive intravaskuläre Sonographie (IVUS) ermöglicht eine hochauflösende Darstellung koronarer Gefäße und bildet derzeit den Gold-Standard zur Beurteilung arteriosklerotischer Gefäßveränderungen [1]. Die Untersuchung erfolgt im allgemeinen unter Verwendung eines Führungskatheters, der über eine Schleuse an der Leistenarterie in das Gefäßsystem eingebracht wird. Durch den Führungskatheter wird ein Ultraschallkatheter, in dem sich ein Hochfrequenz-Transducer befindet, in den Bereich der zu untersuchenden Koronararterie geschoben. Zur Akquisition dreidimensionaler Datensätze in Form tomographischer Bildfolgen wird ein motorgesteuerter Rückzug des Transducers durchgeführt. Eine manuelle Auswertung der dabei aufgenommenen Datensätze ist sehr zeitaufwendig. Die bisher bekannten semi-automatischen Verfahren zur computerunterstützten Analyse [2] können hierbei eine Hilfestellung leisten, weisen jedoch Einschränkungen bezüglich der darstellbaren Konturformen und der Reproduzierbarkeit der Konturbestimmung auf. Automatische Analysetechniken zur reproduzierbaren Segmentierung der umfangreichen Bilddatensätze sind daher zur diagnostischen Beurteilung von StenoseProzessen wünschenswert. Eine Methode zur vollautomatisierten Bestimmung der inneren Gefäßwandkontur ist in [3] beschrieben. Im folgenden wird eine neue Methode zur Bestimmung der inneren und äußeren Gefäßwandkontur (Intima und Media) auf der Basis sagittaler IVUS-Schnittbilder vorgestellt. Die Konturen bilden die Grundlage für die dreidimensionale Rekonstruktion der Oberfläche unter Verwendung eines eigenentwickelten Systems zur 3D-Darstellung intravaskulärer tomographischer Datensätze [4].

\section{METHODE}

Die der Methode zugrundeliegenden dynamischen geometrischen Konturmodelle (DGKM) sind der Klasse der aktiven Konturmodelle zuzuordnen. Mit den snakes wurden in [5] erstmals aktive Konturmodelle beschrieben, bei denen Energieterme zur Steuerung von Splines verwendet wurden. Seitdem wurden vielfältige Varianten und Weiterentwicklungen auf diesem Gebiet realisiert $[6,7]$. Wir verwenden ein diskretes geschlossenes
Konturmodell, bestehend aus Knoten $V_{i}$, die durch Kanten $\mathbf{d}_{i}$ verbunden sind (Abb. 1).
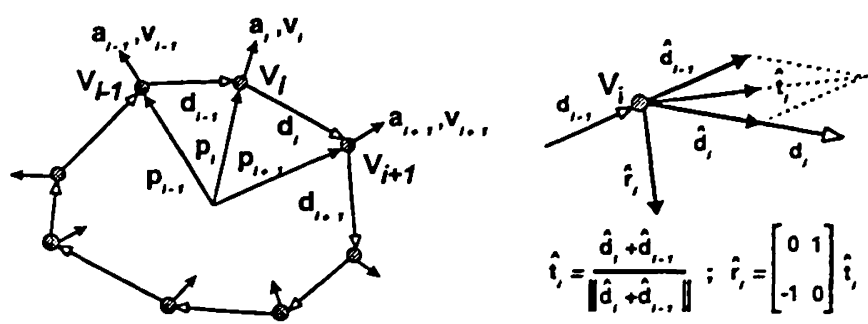

Abb. 1: Dynamisches geometrisches Konturmodell.

Jedem Knoten wird die Masse $m_{i}$ zugeordnet, über die die Trägheit des Modells festgelegt wird. Im ersten Schritt des Verfahrens erfolgt wahlweise eine manuelle oder automatische Initialisierung der Kontur. Die anschließende Verformung der Kontur wird durch innere und äußere Kräfte gesteuert, die an den Knotenpunkten ansetzen. Die zugrundeliegende Lagrange'sche Bewegungsgleichung

$$
\mathbf{f}_{i}=m_{i} \frac{\partial^{2} \mathbf{p}_{i}}{\partial t^{2}}+\gamma_{i} \frac{\partial \mathbf{p}_{i}}{\partial t}=m_{i} \mathbf{a}_{i}+\gamma_{i} \mathbf{v}_{i}
$$

für das dynamische Masseknoten-System beinhaltet eine Dämpfungskonstante $\gamma_{i}$ und wird iterativ unter Verwendung der numerischen Integration nach Euler gelöst.

Die am Knoten $V_{i}$ angreifende resultierende $\mathrm{Kraft}$ ist dabei durch eine mit den Faktoren $\omega_{i n t}$ und $\omega_{\text {ext }}$ gewichtete Summe interner und externer Kräfte sowie der geschwindigkeitsabhängigen Dämpfungskraft $\boldsymbol{f}_{i}^{d}$ gegeben zu

$$
\begin{gathered}
f_{i}^{\text {ges }}=\mathbf{f}_{i}+\mathbf{f}_{i}^{d} \text { mit } \mathbf{f}_{i}=\omega_{i n t} f_{i}^{\text {int }}+\omega_{e x t} f_{i}^{\text {ext }} \\
\text { und } f_{i}^{d}=-\gamma_{i} \mathbf{v}_{i} .
\end{gathered}
$$

Der iterative Anpassungsproze $B$ erfordert neben der Berechnung der Ortsvektoren $\boldsymbol{p}_{i}$, Geschwindigkeiten $\mathbf{v}_{i}$ und Beschleunigungen $\mathbf{a}_{i}$ der Knoten in jedem Iterationsschritt auch eine Neuabtastung der Kontur. Dabei werden Knoten neu eingefügt oder entfernt, sofern eine bestimmte Länge der Kanten $\boldsymbol{d}_{i}$ über- oder unterschritten wird. Der ProzeB terminiert, wenn sich alle Stützstellen der Kontur im Gleichgewichtszustand befinden oder eine benutzerdefinierte Anzahl von Iterationen erreicht ist.

Die inneren Kräfte wirken in Richtung $\mathbf{r}_{i}$ (Abb. 1, rechts) und werden aus der lokalen Krümmung

$$
c_{i}=\hat{d}_{i+1}-\hat{d}_{i} \text { mit } \hat{d}_{i}=\frac{p_{i+1}-p_{i}}{\left\|p_{i+1}-p_{i}\right\|}
$$


der Kontur zu

$$
\mathbf{f}_{i}^{\text {int }}=\left(c_{i} \otimes h\right) \hat{\mathbf{r}}_{i} \text { mit } h=\left[\begin{array}{lll}
-0,5 & 1 & -0,5
\end{array}\right]
$$

berechnet. Die Hochpaßfilterung mittels Faltung $(\otimes)$ dient der Eliminierung von Gleichanteilen zur Vermeidung einer möglichen Schrumpfung des Modells bei konstanter Krümmung. Zur Bestimmung der inneren Konturen wird im Gegensatz zu [6] eine adaptive Ballonkraft eingeführt, die eine Expansion einer automatisch generierten kreisförmigen Initialkontur bewirkt. Diese wird über eine Histogrammauswertung des Originalbildes in einer symmetrischen inneren und äußeren Nachbarschaft der Knoten unter Verwendung der Differenz beider Medianwerte berechnet.

Die äußeren Kräfte werden aus einer über eine Linearkombination mehrerer Bildmerkmale berechneten Energiefunktion

$$
\mathbf{f}_{i}^{e x t}=\left(-\nabla E^{e x t} \cdot \hat{r}_{i}\right) \hat{r}_{i}
$$

abgeleitet. Die lokale Auflösung wird dabei unter $\mathrm{Zu}$ grundelegung einer zweidimensionalen Gaußfilterung

$$
I_{\sigma}^{\delta}(x, y)=G_{\sigma}(x, y) \otimes I(x, y)
$$

des Originalbildes $I(x, y)$ in einem sequentiellen Multiresolution-Verfahren bei abnehmendem Wert der Standardabweichung optimiert. Zur Bestimmung der inneren Kontur eignet sich die Energiefunktion

$$
E_{i k}^{e x t}=\omega_{i m} I_{\sigma}^{g}(x, y)+\omega_{e d s e}\left\|\nabla I_{\sigma}^{\delta}(x, y)\right\|
$$

In die Energiefunktion

$$
E_{A K}^{e x t}=\omega_{i m} I_{\sigma}^{\delta}(x, y)+\omega_{M H}\left\|\Delta J_{\sigma}^{\delta}(x, y)\right\|
$$

zur Detektion der linienförmigen äußeren Kontur wurde die Berechnung eines modifizierten Marr-HildrethOperators miteinbezogen [5].

Die Konturdatensätze bilden die Grundlage für eine auf diesem Gebiet neue, aussagekräftige und realzeitfähige Form der dreidimensionalen Oberflächendarstellung, die speziell zur Visualisierung intravaskulärer tomographischer Datensätze entwickelt wurde. In Abbildung 2 sind zwei Rekonstruktionen arteriosklerotisch veränderter koronarer Gefäßsegmente dargestellt.

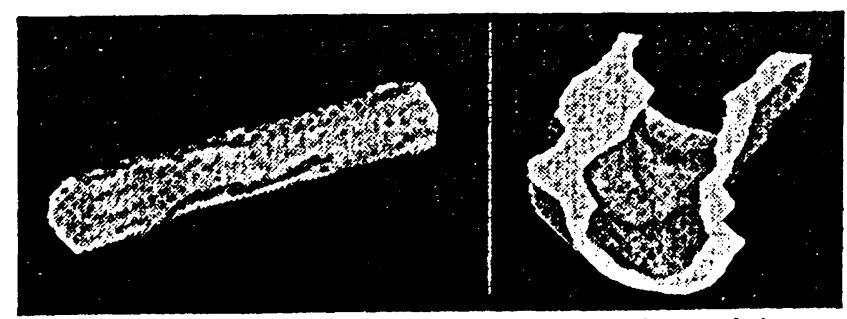

Abb. 2: DGKM-basierte 3D-Oberflächenrekonstuktionen.

\section{ERGEBNISSE}

In vier, jeweils 75 Einzelbilder umfassenden Bildsequenzen erfolgte eine automatische Konturbestimmung mit anschließendem stichprobenhaften Flächenvergleich mit
Konturen, die zwei Ärzte unter Verwendung eines Touchscreen-Monitors einzeichneten (Abb. 3).
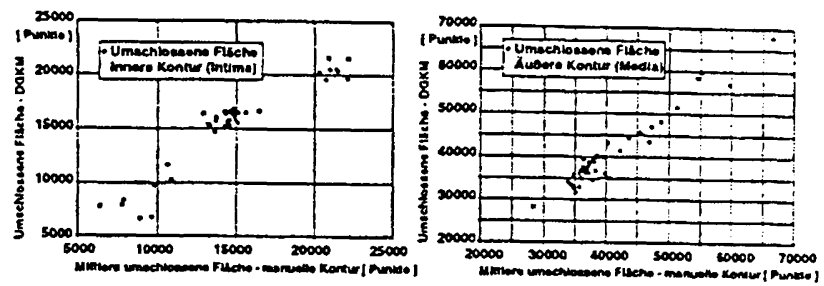

Abb. 3: Flächenvergleich automatisch generierter innerer (links) und äußerer Konturen (rechts) mit mittleren manuellen Werten.

Die durchschnittliche Rechenzeit zur Doppelkonturbestimmung lag bei Bildern mit $256 \times 256$ Pixeln und einer Auflösung von 8 Bit bei $90 \mathrm{~s} / \mathrm{Bild}$ (Pentium, $166 \mathrm{MHz}$ ).

\section{ZUSAMMENFASSUNG}

Das vorgestellte dynamische geometrische Konturmodell ist im Gegensatz zu Modellen, die eine radiale Abtastung zugrunde legen, weit weniger eingeschränkt bezüglich der darstellbaren Formen und ermöglicht daher auch eine Erfassung komplex geformter Läsionen. Da das Konturmodell geschlossen ist, können Lücken im Konturverlauf überbrückt werden. Die bisherigen Untersuchungen ergeben eine gute Übereinstimmung mit Konturen, die von Ärzten manuell eingezeichnet wurden. Aufgrund der Vielzahl möglicher Einstellungen der Ultraschallgeräte muß ein einmaliger Abgleich der Parameter des Segmentierungsverfahrens erfolgen. Ein genereller Einsatz erfordert die weitere Evaluierung unter zusätzlicher Einbeziehung von in vitro-Untersuchungen.

\section{LITERATUR}

[1] Görge, G.: Intrakoronarer Ultraschall: Der ncue Gold-Standard? 63. Jahrestagung der Deutschen Gesellschaft für Kardiologie, 1997.

[2] Birgelen, C., et al.: Morphometric analysis in threcdimensional intracoronary ultrasound. American Heart Journal 2, S. 516-527, 1996.

[3] Schmauder, M., Gross, C. M., et al.: Automated detection of endovascular contours from intravascular ultrasound image sequences using a simulated annealing approach. VIII Mediter. Conf. on Medical and Biol. Engineering and Computing, Cyprus 1998.

[4] Schmauder, M., Orglmeister, R., et al.: 3D-Obertlachenrekonstruktion koronarer Gefäßwände unier Verwendung segmentierter intravaskulärer Uliriaschalldatensätze. 2. Symposium Quantitatici Sonographie, Halle 1998.

[5] Kass, M.. et al.: Snakes: Active Contour Models. Int. Journal of Computer Vision, 1, 321-331, $198 \mathrm{~s}$.

[6] Cohen, L.: On Active Contour Models and Ballons CVGIP: Image Understanding, 53, 211-218. 1991.

[7] Lobregt, S., Viergever, A.: Discrete Dynamis Contour Model. IEEE Transactions on Mcdicial Imaging, 14, 12-24, 1995. 